\title{
Das Impfdilemma
}

\section{Noa Kropf}

Medizinstudentin Universität Zürich

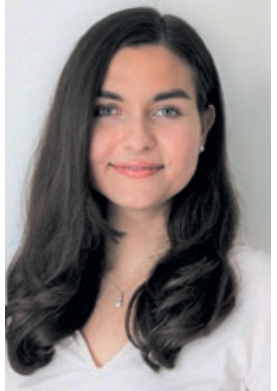

Es ist ein regnerischer Montag, welcher sich langsam dem Ende zuneigt. An der Universitätsstrasse Zürich befinden sich die Eltern der dreijährigen Maya Hefti (Name geändert) im obersten Stock der Uniklinik. Die Ärztin Susanna Lindner hat sie soeben über die Masernimpflücke ihrer Tochter informiert. Die Eltern sind gar nicht gut darauf zu sprechen. Aufgrund ihrer Weltanschauung lehnen sie jegliche Impfungen ab. Auf den Gesichtern der Eltern ist Unverständnis, wenn nicht sogar leichter Zorn zu sehen. Der Vater erklärt ungeduldig, dass ihrem Weltbild nach eine solche Impfung als verpönt angesehen werde. Ein Ausschluss aus der Gesellschaft, soziale Isolation und die Verachtung Gottes wären die Folge für die gesamte Familie. Während der Pause berichtet die Pflegefachfrau mir, Medizinstudentin im ersten Jahr, von einem ähnlichen Fall, der sich vor zwei Jahren ereignet hatte. Die Eltern eines fünfjährigen Jungen hätten sich aus Glaubensgründen geweigert, ihren Sohn impfen zu lassen - was dann aber trotzdem geschehen war. Ein Jahr später sei der Vater Alkoholiker geworden, die Mutter wurde daraufhin in die Psychiatrie eingeliefert. Sie seinen komplett aus dem eigenen sozialen Umfeld ausgeschlossen worden, alle sozialen Kontakte der Familie seien vom einen Tag auf den anderen zunichte gewesen. Der Vater hätte ein Jahr später Suizid begangen. Seither lebte die Mutter mit dem Kind alleine, ihr ginge es immer noch nicht gut.

Nach diesem Gespräch begebe ich mich auf den Heimweg. Und obwohl ich nicht gefragt bin, identifiziere ich mich mit der Ärztin, und die Entscheidung trifft mich stark. Ich merke, wie ich jetzt mit dieser Situation an ihrer Stelle überfordert wäre. Ein Dilemma, welches ich eher aus passiver Sicht aus dem Kino kenne, scheint jetzt plötzlich Realität geworden zu sein. Wie würde ich an dieser Stelle handeln? Im Tram nach Hause fällt es mir schwer, mir meiner Intuition bewusst zu werden. Die Worte der Pflegefachfrau gehen mir nicht aus dem Kopf. Ich merke, wie ich unsicher darüber bin, wie sich die möglichen Folgen der Impfverweigerung auf das Leben des Kindes auswirken könnten. Die Unsicherheit wandelt sich in ein Gefühl der Angst. Was geschieht, wenn es der Familie Hefti genauso ergehen würde wie der Familie aus dem vorherigen Fall? Wäre dies das Ende ihres Familienlebens? Lohnt es sich, den sozialen Ausschluss zu riskieren, um die Ansteckungsgefahr einer Krankheit zu eliminieren, die nicht allzu häufig zu sein scheint? Wo bliebe ausserdem im Falle einer Impfung die Autonomie der betreffenden Personen? Wäre nicht das Selbstbestimmungsrecht verletzt, wenn man eine Impfung erzwingen würde?

Ich überlege mir aber auch, ob die Verweigerung überhaupt dem Willen des Kindes selbst entspricht. Denn Maya hat sich noch nicht aus eigenem Willen für die Religion entschieden und dafür, dass sie nach diesen Gesetzen leben will. Was wäre also, wenn sie sich in ein paar Jahren gar nicht mit dem Glauben der Eltern identifizieren kann, aber an Masern erkrankt? Sie würde dann ein Opfer bringen für eine Religion, welcher sie sich gar nicht angehörig fühlt. Viele Fragen gehen mir durch den Kopf. Mir wird bewusst, wie viel Informationen über die familiäre Situation und das soziale Umfeld mir fehlen. Da ist es schwierig, sich Gedanken zu machen und abzuwägen.

Ich überlege weiter, ob es nicht das Recht jedes Elternteils ist, frei über die Religionsausübung des eigenen Kindes zu entscheiden, bis dieses selber dazu in der Lage ist. So werden im Judentum und Islam Kinder in frühem Alter beschnitten, ohne nach ihrer Einwilligung zu fragen. Wie sehen aber die Gefahren einer Beschneidung im Vergleich zu jenen einer Masernimpfung aus? Ich merke schnell, dass es in dieser Situation schwer ist, einen Referenzpunkt, einen Massstab oder eine vergleichbare Situation zu finden. Ich erkenne den Kern des Problems: Für ein ethisches Dilemma gibt es keine Lösung nach Rezept, doch ein Arzt muss dennoch eine tragbare Lösung finden.

In diesem Moment öffnen sich die Tramtüren, und ein Kinderwagen wird in das Tram geschoben. Darin sitzt ein Kleinkind mit einem roten Ausschlag auf dem Gesicht. Zwischen der gereizten Haut stechen die blauen Augen des Kindes hervor. Die Mutter steht nun auch im Tram. Sie sieht ausgelaugt aus, fast schon leidend. Ich schlucke. Ich merke, wie mein Wissen über den vorherigen Fall und die möglichen sozialen Konsequenzen für die Familie meine Gedanken von der Krankheit selber abgelenkt haben. Was ist, wenn das Kind nicht geimpft wird, mit Masern angesteckt wäre und dann weitere nicht geimpfte Kinder im Hort ansteckt? Eine Masernepidemie wäre die Folge. Sofort ziehe ich das Handy aus meiner Tasche und recherchiere die möglichen Konsequenzen. Ich lese von einer Masernepide- 
mie, welche im Jahr 2000 in New York ausgebrochen ist, obwohl die Krankheit bereits als ausgerottet galt. Antiimpfkampagnen, welche davor stattgefunden hätten, seien die Ursache für diese Epidemie gewesen.

Bei einem Masernfall in einem Hort wird dem kranken Kleinkind verboten, den Hort zu besuchen. Für die Eltern heisst das, dass sie sich bei der Arbeit einige freie Tage nehmen müssen. So kann sich also ein vereinzelter Masernfall auf den Berufsalltag von mehreren Eltern auswirken. Ein solcher Massenausbruch liesse sich aber nur verhindern, wenn schätzungsweise $95 \%$ der Bevölkerung immun sind, lese ich weiter. Bei mehreren einzelnen Fällen von Nachlässigkeit gäbe es also die Gefahr eines Dominoeffekts, und das Risiko einer solchen Epidemie würde drastisch steigen. Denn wo soll eine Grenze gezogen werden, wenn man bereits mehrere Ausnahmen gemacht hat?

Ausserdem lese ich, dass die Krankheit zu einer Gehirnhautentzündung führen kann. Sogar Lähmung und Behinderung könnten mögliche Konsequenzen sein. Weiter lese ich, dass Kleinkinder in Mayas Alter zu einer Risikogruppe gehören und an Komplikationen leiden könnten, wenn sie sich einmal angesteckt haben.

Der Gedanke einer Epidemie trifft mich in dieser Zeit, der Zeit der Coronakrise, stark. Würde die Ärztin bei Verzicht auf die Impfung womöglich eine potentielle Patientin 0 kreieren? Die Folgen einer Epidemie durfte ich mit meiner Familie und der gesamten Welt gerade erst mit eigenen Augen beobachten und miterleben. Die Einstellung aller sozialen Kontakte, die Eindämmung der Wirtschaft sowie einer damit einhergehenden steigenden Arbeitslosigkeit waren einige indirekte Folgen des Lockdowns. Wäre der Verzicht auf die Impfung also nicht eine Handlung gegen das allgemeine Wohl? Im schlimmsten Falle würden tausende von Menschen darunter leiden. Ist eine Impfverweigerung also nicht sogar egoistisch?

Ich realisiere schnell, dass diese Entscheidung nicht nur Maya und ihre Eltern betrifft. Die Konsequenzen der Entscheidung können im schlimmsten Fall eine ganze Bevölkerung treffen. So entpuppt sich die Entscheidung über die Impfung als ein tiefgreifendes ethisches Dilemma. Ein Dilemma, welches sich in einem
Konflikt zwischen Religion und Meinungsfreiheit, Gesundheit, aber auch Ökonomie begründet. Zum Glück realisiere ich im nächsten Moment, dass ich die Entscheidung noch nicht treffen muss, und bin froh. Aber obgleich ich jetzt noch nicht gefragt bin, werde ich es in Zukunft sein.

Eine Woche später bin ich wieder im Spital. Es hat bereits ein Gespräch unter Ärzten und Ethikern stattgefunden und die Entscheidung ist gefallen. Gespannt warte ich auf die Pflegefachfrau, die mir schliesslich erzählt, dass die Impfung am vorherigen Freitag durchgeführt worden sei. Ich frage direkt nach, wie die Eltern reagiert hätten und ob man seither etwas von der Familie gehört habe. In dem Moment betritt Frau Hefti, Mayas Mutter, die Krankenhausetage. Mit Tränen in den Augen begegnet sie mir und der Pflegefachfrau. Eine Spielgruppenfreundin Mayas, deren Eltern auch Mitglieder derselben religiösen Gruppierung seien, sei an Masern erkrankt. Sie wolle die Ärztin sprechen und sich für ihre Hartnäckigkeit bedanken. Schliesslich kommt die Ärztin und verschwindet mit Frau Hefti im Zimmer.

Plötzlich bin ich mir sicher, dass das Richtige getan wurde. Ein gutes Gefühl steigt in mir auf, die ganze Anspannung der vorherigen Woche, welche sich in mir aufgestaut hatte, löst sich auf einmal in Luft auf. Ich bin erleichtert und fühle mich zufrieden. Die Ärztin muss jetzt bestimmt ein Gefühl der Tugend verspüren. Doch kaum kommt mir der Gedanke, meldet sich eine andere Stimme in meinem Kopf. «Das war erst der Anfang, es warten noch viele ähnliche Entscheidungen auf dich", muss ich mir eingestehen. Und ich realisiere im selben Moment auch, dass es genug Situationen geben wird, in welchen ich nicht sicher sein werde und mit der Ungewissheit werde leben müssen, ob ich das Richtige oder Falsche getan habe. Ich sehe, welche Herausforderungen von grosser Wichtigkeit und Verantwortung auf mich warten. Bei diesem Gedanken läuft mir ein kalter Schauer über den Rücken. Nichtsdestotrotz wird dieses Gefühl des Respekts begleitet von einem Gefühl der Aufregung und Lust auf die Zukunft. Denn schliesslich ist das genau der Weg, für den ich mich entschieden habe. 